Pacific Journal of Mathematics

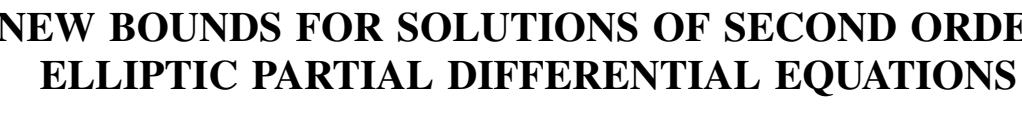




\title{
NEW BOUNDS FOR SOLUTIONS OF SECOND ORDER ELLIPTIC PARTIAL DIFFERENTIAL EQUATIONS
}

\author{
L. E. Payne and H. F. Weinberger
}

1. Introduction In a previous paper [10] the authors presented methods for determining, with arbitrary and known accuracy, the Dirichlet integral and the value at a point of a solution of Laplace's equation. These methods have the advantage that upper and lower bounds are computed simultaneously. Moreover all error estimates are in terms of quadratic functionals of an arbitrary function, so that the Rayleigh-Ritz technique gives a systematic way of making the error arbitrarily small. These methods depend on an identity of F. Rellich [12]. As a consequence it is necessary to assume that the boundary is star-shaped with respect to some point, and it is not possible to treat differential equations with variable coefficients by these methods.

In this paper a generalization of Rellich's identity to general second order elliptic operators as well as to a large class of elliptic systems of second order operators is employed to extend the results of the previous paper to equations involving such operators and rather general domains.

The identity in question was obtained and used for hyperbolic operators by L. Hörmander [7] who, in a mimeographed note kindly communicated to the authors, has independently obtained therefrom some estimates for boundary values of the solution of a second order elliptic equation.

It is interesting to note the similarity in structure of the identity (2.4) and the formula for the first variation of Green's function for $\Delta u-p u$ given by Garabedian and Schiffer [6].

For the sake of simplicity only the case of a self-adjoint second order operator without zero order terms is treated in detail. However the method is easily extended to more general operators, and even systems, as is shown in $\S \S 6$ and 7 .

Section 2 is concerned with the above-mentioned identity. In $\S 3$ this identity is used to estimate several important quadratic functionals (including the generalized Dirichlet integral) in terms of Dirichlet data for a general non-homogeneous boundary value problem in $N$ dimensions. We obtain an approximation to the generalized Dirichlet integral by means of a specific functional of an arbitrarily chosen function. The error estimate is a quadratic functional in the deviation of the Dirichlet data of the arbitrary function from the given data, and can be made

Received May 15, 1958. This research was supported by the United State Air Force through the Air Force Office of Scientific Research of the Air Research and Development Command under contract No. AF 18(600)-573. 
small by a Rayleigh-Ritz technique. The previous paper [10] treated only Laplace's equation of which many solutions are known, so that the arbitrary function could be assumed to satisfy the differential equation. Here, however, a general differential equation is treated. Therefore provision must be made for errors of the arbitrary function both in the differential equation and in the boundary values, and the error estimate is a quadratic functional in both of these. This estimate can be useful even in the case of the Laplace operator when the domain or the Dirichlet data are such that it is inconvenient to work with harmonic functions.

Even in the special case treated in the previous paper, that is, when the equation is Laplace's equation, the domain is star-shaped, and the arbitrary function is taken to be harmonic, the estimate (3.29) represents an improvement over the estimate (3.15) of [10]. The same statements apply to $\S 4$, where the value of the solution of a Dirichlet problem at an arbitrary interior point is given.

In $\$ 5$ similar estimates are derived for the exterior mixed boundary value problem in three or more dimensions. Here the inequalities (5.10) and (5.11) are basic, both to the estimate of the Dirichlet integral and to the pointwise approximation of the function. In those Neumann problems which could be treated in the previous paper, the present method represents a great simplification. In fact, the treatment of Neumann's problem is rendered simpler than that of Dirichlet's problem. Unfortunately, the occurrence of a zero eigenvalue prevents our results from being extended to the interior Neumann problem.

Section 6 sketches the extension of all these results to non-selfadjoint second order equations. In $\S 7$ these extensions are carried to a class of elliptic systems of second order differential equations. This extension permits the treatment of the first boundary value problem, the exterior second boundary value problem, as well as certain mixed problems of the classical theory of elasticity [13].

The treatment of the mixed boundary value problems is an extension of the results of the report [11] upon which this paper is based.

\section{An integral identity. We consider the operator}

$$
\mathfrak{A}(u)=\left(a^{i j} u,{ }_{, i}, \quad x=\left(x^{1}, \cdots, x^{N}\right)\right.
$$

on a domain $D$ of Euclidean $N$-space. The summation convention will be used throughout this paper. The symbol ${ }_{, i}$ indicates partial differentiation with respect to $x^{i}$. The components of the symmetric matrix $a^{i j}(x)$ are taken to be piecewise continuously differentiable functions in $D$. We assume the existence of two positive constants $a_{0}$ and $a_{1}$, such that for all numbers $\left(\xi_{1}, \cdots, \xi_{N}\right)$ and all $x$ in $D$

$$
a_{0} \sum_{i=1}^{N} \xi_{i}^{2} \leqq a^{i j} \xi_{i} \xi_{j} \leqq a_{1} \sum_{i=1}^{N} \xi_{i}^{2} .
$$


It can be shown by direct differentiation that for any piecewise continuously differentiable functions $f^{1}(x), \cdots, f^{N}(x)$

$$
\begin{aligned}
\left(\left[f \mathrm{fa}^{i j}-f^{i} a^{k j}-f^{j} a^{i k}\right] u_{, i} u_{, j}\right)_{, k}+2 f^{i} u_{, i} \mathfrak{Q}(u) \\
=\left[f_{, k}^{k} a^{i j}-f_{, k}^{i} a^{k j}-f_{, k}^{j} a^{i k}+f^{k} a_{, k}^{i j}\right] u_{, i} u_{, j} .
\end{aligned}
$$

We integrate this identity over $D$ and apply the divergence theorem to the left-hand side. The result is

$$
\begin{aligned}
\oint_{B}\left[f^{k} a^{i j}-f^{i} a^{k j}-\right. & \left.f^{j} a^{i k}\right] u,{ }_{, \imath} u,{ }_{, j} n_{k} d S=-\iint_{D} f^{i} u, i \mathfrak{U}(u) d V \\
& +\iint_{D}\left[f_{, k}^{k} a^{i j}-f_{, k}^{i} a^{k j}-f{ }_{, k} a^{i k}+f^{k} a_{, k}^{i j}\right] u_{, i} u,{ }_{, j} d V .
\end{aligned}
$$

Here $B$ is the boundary of $D, n_{k}$ is a Euclidean unit outward normal vector $\left(\sum_{k=1}^{N} n_{k}^{2}=1\right)$, and $d S$ and $d V$ are Euclidean elements of area and volume.

An identity equivalent to (2.4) has been given by Hörmander [7]. In the special case where $\mathfrak{U}(u)$ is the Laplace operator and $f^{i}=x^{i}$, the identity reduces to that of Rellich [12].

The usual Green's identity for $2(u)$ is

$$
\iint_{D} u \mathfrak{A}(u) d V+A[u]=\oint_{B} u \frac{\partial u}{\partial \nu} d S,
$$

where we define the generalized Dirichlet integral

$$
A[u]=\iint_{D} a^{i j} u_{, i} u, j d V
$$

and the conormal derivative

$$
\frac{\partial u}{\partial \nu}=a^{i j} u_{, i} n_{j} .
$$

We decompose the derivatives of $u$ appearing in the boundary integral of (2.4) into conormal and tangential derivatives. To this effect, we note that the vector

$$
\mathrm{T}^{j}=a^{i_{i}}\left[u_{, i}-\frac{\partial u}{\partial \nu}\left(n / a^{\prime q} n_{p} n_{q}\right)\right]
$$

is orthogonal to $n_{j}$, and hence is a tangent vector. In fact, it is the projection into the tangent plane of the contravariant gradient of $u$. We introduce the inverse matrix $a_{i j}$ of $a^{i j}$, and renormalize by setting

$$
t^{i}=\mathrm{T}^{i}\left[\begin{array}{l}
a^{p q} n_{p} n_{q} \\
a_{r s} \mathrm{~T}^{r} \mathrm{~T}^{s}
\end{array}\right]^{1 / 2}
$$


so that

$$
a_{i j} t^{i} t^{j}=a^{i j} n_{i} n_{j}=n
$$

(This defines the function $n$ on $B$ ).

The directional derivative

$$
\frac{\partial u}{\partial t}=u_{, i} t^{i}
$$

is in a tangential direction. Furthermore it follows from (2.8) and (2.9) and the fact that $u, \mathrm{~T}^{j}=a_{i j} \mathrm{~T}^{i} \mathrm{~T}^{j}$ that

$$
a^{i j} u_{, i} u_{, j}=n^{-1}\left[\left(\frac{\partial u}{\partial \nu}\right)^{2}+\left(\frac{\partial u}{\partial t}\right)^{2}\right]
$$

and

$$
f^{i} u_{, i}=n^{-1}\left[f^{i} n_{i} \frac{\partial u}{\partial \nu}+a_{i j} f^{i} t^{j} \frac{\partial u}{\partial t}\right]
$$

Then (2.4) may be written as

$$
\begin{aligned}
& \oint_{B} n^{-1}\left[f^{k} n_{k}\left\{\left(\frac{\partial u}{\partial t}\right)^{2}-\left(\frac{\partial u}{\partial \nu}\right)^{2}\right\}-2 a_{i j} f^{i} t^{j} \frac{\partial u}{\partial \nu} \frac{\partial u}{\partial t}\right] d S \\
& =-2 \iint_{D} f^{i} u_{, i} \mathfrak{I}(u) d V+\iint_{D}\left[f_{, k}^{k} a^{i j}-f_{, k}^{i} a^{j k}-f_{, k}^{j} a^{i k}+f^{k} a_{, k}^{i j}\right] u_{, i} u{ }_{, j} d V .
\end{aligned}
$$

This identity is fundamental to our estimates in the Dirichlet problem.

3. Bounds for quadratic functions in Dirichlet's problem.

(a) Homogeneous boundary conditions.

Let $u(x)$ be a function satisfying

$$
u=0 \quad \text { on } B \text {, }
$$

with $\mathfrak{A}(u)$ given in $D$.

We first bound the generalized Dirichlet integral $A[u]$ defined by (2.6). Noting that the right-hand side of (2.5) vanishes, we have by Schwarz's inequality

$$
(A[u])^{2} \leqq \iint_{D} u^{2} d V \iint_{D}[\mathfrak{Y}(u)]^{2} d V
$$

In order to bound the integral of $u^{2}$ we use the lowest eigenvalue $\lambda_{1}$ of the fixed membrane problem 


$$
\begin{aligned}
\Delta \varphi+\lambda \varphi=0 & & \text { in } D \\
\varphi=0 & & \text { on } B .
\end{aligned}
$$

It is well known [1] that since $u=0$ on $B$.

$$
\iint_{D} u^{2} d V \leqq \frac{1}{\lambda_{1}} \iint_{D} \sum_{l=1}^{N}\left(u_{, i}\right)^{2} d V
$$

Thus, from (2.2) it follows that

$$
\iint_{D} u^{2} d V \leqq \frac{1}{a_{0} \lambda_{1}} A[u]
$$

Hence, by (3.2) we find the bound

$$
A[u] \leqq \frac{1}{a_{0} \lambda_{1}} \iint_{D}[\mathfrak{U}(u)]^{2} d V .
$$

From (3.5) we also have the bound

$$
\iint_{D} u^{2} d V \leqq \frac{1}{a_{0}^{2} \lambda_{1}^{2}} \iint_{D}[\mathfrak{U}(u)]^{2} d V
$$

A lower bound for $\lambda_{1}$ is easily obtained. For example the isoperimetric inequality of Faber [4] and Krahn [9] states that $\lambda_{1}$ is at least as large as the lowest fixed membrane eigenvalue of a sphere of the same volume as that of $D$.

We now seek a bound for $\oint_{B}(\partial u / \partial \nu)^{3} d S$. By (3.1) $\partial u / \partial t=0$ on $\mathrm{B}$, and the left-hand side of (2.14) reduces to a single term.

We choose the vector field $f^{k}$ in such a way that $f^{k} n_{k}$ is bounded and has a positive minimum on $B$. For example, if $B$ is star-shaped with respect to the origin, one can take $f^{k}=x^{k}$. If $D$ is a region between two surfaces that are star-shaped with respect to the origin, and if $D$ contains the spherical shell of radius $r_{0}$, one can take $f^{k}=x^{k}\left(r-r_{0}\right)$.

Because of the fact that $a^{i j}$ is positive definite, there exists a constant $c$ such that

$$
-\left[f_{, k}^{k} a^{i j}-f_{, k}^{i} a^{j k}-f_{, k}^{j} a^{i k}+f^{k} a_{, k}^{i j}\right] u_{, i} u,{ }_{, j} \leqq c a^{i j} u_{, i} u, j
$$

throughout $D$. ( $c$ is any upper bound for the largest eigenvalue of the coefficient matrix on the left with respect to $a^{i j}$ ).

From (2.14) together with (2.5) and (3.8) follows the inequality

$$
\oint_{B} n^{-1} f^{k} n_{k}\left(\frac{\partial u}{\partial \nu}\right)^{2} d S \leqq \iint_{D}\left[2 f^{i} u_{, i}-c u\right] 2(u) d V .
$$

Thus by Schwarz's inequality we have 


$$
\left[\oint_{B} n^{-1} f^{k} n_{k}\left(\frac{\partial u}{\partial \nu}\right)^{2} d S\right]^{2} \leqq \iint_{D}\left[2 f^{i} u, i-c u\right]^{2} d V \iint_{D}[\mathfrak{U}(u)]^{2} d V
$$

Application of the triangle inequality and Schwarz's inequality as well as (3.6) and (3.7) yields

$$
\begin{aligned}
\left\{\iint_{D}\left[2 f^{i} u_{, i}-c u\right]^{2} d V\right\}^{1 / 2} & \leqq 2 \max \left[a_{i j} f^{i} f^{j}\right]^{1 / 2}(A[u])^{1 / 2}+c\left[\iint_{D} u^{2} d V\right]^{1 / 2} \\
\leqq & \left\{2 \max \frac{\left[a_{i j} f^{i} f_{j}\right]^{1 / 2}}{\left(a_{0} \lambda_{1}\right)^{1 / 2}}+\frac{c}{a_{0} \lambda_{1}}\right\}\left\{\iint_{D}[\mathfrak{U}(u)]^{2} d V\right\}^{1 / 2} .
\end{aligned}
$$

If we first integrate the left-hand side of (3.11) by parts and then apply the same inequalities, we arrive at an inequality similar to (3.11) but with the first factor of the second line on the right replaced by

$$
\left[4 \max \frac{a_{i j} f^{i} f^{i}}{a_{0} \lambda_{1}}+c \frac{\left(2 \max f_{, i}^{i}+c\right)}{a_{0}^{2} \lambda_{1}^{2}}\right]^{1 / 2} .
$$

Thus we have

$$
\oint_{B} n^{-1} f^{k} n_{k}\left(\frac{\partial u}{\partial \nu}\right)^{2} d S \leqq K \iint_{D}[\mathfrak{A}(u)]^{2} d V
$$

where

$$
\begin{array}{r}
K=\frac{1}{a_{0} \lambda_{1}}\left[4 \max \left(a_{\iota j} f^{i} f^{j}\right) a_{0} \lambda_{1}+c^{2}+2 c \inf \left\{\max f_{, i}^{i},\right.\right. \\
\left.\left.2\left[a_{0} \lambda_{1} \max \left(a_{i j} f^{i} f^{\jmath}\right)\right]^{1 / 2}\right\}\right]^{1 / 2} .
\end{array}
$$

Hence we have finally

$$
\oint_{B}\left(\frac{\partial u}{\partial \nu}\right)^{2} d S \leqq \frac{K}{\min \left(n^{-1} f^{k} n_{k}\right)} \iint_{D}[\mathfrak{L}(u)]^{2} d V .
$$

(b) Homogeneous differential equation.

Let $v(x)$ be a solution of

$$
\mathfrak{U}(v)=0 \text { in } D
$$

and let $v$ be a given piecewise continuously differentiable function on $B$.

Upon use of (3.8) and (2.5) the identity (2.14) gives

$$
\text { (3.16) }-\oint_{B} n^{-1}\left[f^{k} n_{k}\left\{\left(\frac{\partial v}{\partial t}\right)^{2}-\left(\frac{\partial v}{\partial \nu}\right)^{2}-2 a_{i j} f^{i} t^{j} \frac{\partial v}{\partial \nu} \frac{\partial v}{\partial t}\right] d S \leqq c \oint_{B} v-\frac{\partial v}{\partial \nu} d S\right. \text {. }
$$

We again choose $f^{k}$ in such a way that $f^{k} n_{k}$ has a positive lower bound on $B$. Completing the square in (3.16) yields 


$$
\begin{aligned}
& \oint_{B} n^{-1} f^{k} n_{k}\left[\frac{\partial v}{\partial \nu}+\frac{a_{i j} f^{i} t^{j}}{f^{k} n_{k}} \frac{\partial v}{\partial t}-\frac{c n v}{2 f^{k} n_{k}}\right]^{2} d S \\
& \quad \leqq \oint_{B} n^{-1}\left[f^{k} n_{k}\left(\frac{\partial v}{\partial t}\right)^{2}+\frac{1}{f^{k} n_{k}}\left(a_{i j} f^{i} t^{j} \frac{\partial v}{\partial t}-\frac{c n v}{2}\right)^{2}\right] d S .
\end{aligned}
$$

In order to estimate $A[v]$ we rewrite the Green's identity (2.5) as

$$
\begin{gathered}
A[v]+\oint_{B} \frac{v}{f^{k} n_{k}}\left(a_{i j} f^{i} t^{j} \frac{\partial v}{\partial t}-c n v\right) d S \\
=\oint_{B} v\left[\frac{\partial v}{\partial v}+\frac{a_{i j} f^{i} t^{j} \frac{\partial v}{\partial t}-\frac{c n v}{2}}{f^{k} n_{k}}\right] d S .
\end{gathered}
$$

Applying Schwarz's inequality and (3.17) yields

$$
\begin{aligned}
& \left\{A[v]+\oint_{B} \frac{v}{f^{k} n_{k}}\left(a_{i j} f^{i} t^{j} \frac{\partial v}{\partial t}-\frac{c n v}{2}\right) d S\right\}^{2} \\
& \quad \leqq \oint_{B} \frac{n v^{2}}{f^{k} n_{k}} d S \oint_{B} \frac{\left[\left(f^{k} n_{k} \frac{\partial v}{\partial t}\right)^{2}+\left(a_{i j} f^{i} t^{j} \frac{\partial v}{\partial t}-\frac{c n v}{2}\right)^{2}\right]}{n f^{k} n_{k}} d S .
\end{aligned}
$$

All the quantities other than $A[v]$ occurring in this inequality can be computed and are small if the given boundary value of $v$ and its gradient are small in a mean square sense.

In order to compute the quantities $(\partial v / \partial t)^{2}$ and $a_{i j} f^{i} t^{j} \partial v / \partial t$, we recall that they depend only on the given values of $v$ on $B$. Let $\bar{v}$ be a function defined in a neighborhood of $B$, and coinciding with $v$ on $B$ (that is, an extension into $D$ of the given boundary values). Then by the definitions (2.12) and (2.13)

$$
\begin{aligned}
(\partial v / \partial t)^{2} & =(\partial \bar{v} / \partial t)^{2}=n a^{i j} \bar{v}_{, i} \bar{v}_{, j}-\left(\frac{\partial \bar{v}}{\partial \nu}\right)^{2} \\
a_{i j} f^{i} t^{j} \frac{\partial v}{\partial t} & =n f^{i} \bar{v}_{, i}-f^{i} n_{i} \frac{\partial \bar{v}}{\partial \nu} .
\end{aligned}
$$

(c) Approximation in the general problem.

Let $w$ be the solution of an arbitrary Dirichlet problem. That is, $\mathfrak{H}(w)$ is given in $D$ and $w$ is a given piecewise continuously differentiable function on the boundary $B$.

We approximate $w$ by a twice differentiable function $\varphi$ in the sense that $\mathfrak{A}(\varphi)$ approximates $\mathfrak{A}(w)$ and the boundary values of $\varphi$ approximate those of $w$. We shall show how to estimate $A[w]$ from a knowledge of the function $\varphi$ and the degree of approximation of the given data. 
The triangle inequality states that

(3.21) $\{A[\varphi]\}^{1 / 2}-\{A[w-\varphi]\}^{1 / 2} \leqq\{A[w]\}^{1 / 2} \leqq\{A[\varphi]\}^{1 / 2}+\{A[w-\varphi]\}^{1 / 2}$.

Since $A[\varphi]$ can be computed explicitly, it is only necessary to bound $A[w-\varphi]$. It follows from the existence theorem for the Dirichlet problem that one can write $w-\varphi$ in the form

$$
w-\varphi=u+v
$$

where

$$
u=0 \quad \text { on } B
$$

and

$$
\mathfrak{A}(v)=0 \text { in } D \text {. }
$$

Furthermore,

$$
v=w-\varphi \text { on } B
$$

and

$$
\mathfrak{A}(u)=\mathfrak{A}(w)-\mathfrak{A}(\varphi) \text { in } D \text {. }
$$

These quantities are known explicitly. It follows from (2.5) and Green's second identity

$$
\iint_{D}[u \mathfrak{A}(v)-v \mathfrak{A}(u)] d V=\oint_{B}\left[u \frac{\partial v}{\partial \nu}-v \frac{\partial u}{\partial \nu}\right] d S
$$

that

$$
A[w-\varphi]=A[u]+A[v] .
$$

Hence we need only use the estimates (3.6) and (3.19) together with (3.25) and (3.26) to find

$$
\text { (3.29) } \begin{aligned}
& A[w-\varphi] \leqq \frac{1}{a_{0} \lambda_{1}} \iint_{D}[\mathfrak{U}(w)-\mathfrak{U}(\varphi)]^{2} d V \\
+ & \left\{\oint_{B} \frac{n(w-\varphi)^{2}}{f^{k} n_{k}} d S\right. \\
\times & \left.\oint_{\boldsymbol{B}} \frac{\left(\left[f^{k} n_{k}-\frac{\partial}{\partial t}(w-\varphi)\right]^{2}+\left[a_{i j} f^{i} t^{j} \frac{\partial}{\partial t}(w-\varphi)-\frac{c n(w-\varphi)}{2}\right]^{2}\right)}{n f^{k} n_{k}} d S\right\}^{1 / 2} \\
& -\oint_{B} \frac{(w-\varphi)}{f^{k} n_{k}}\left[a_{i j} f^{i} t^{\jmath} \frac{\partial}{\partial t}(w-\varphi)-\frac{c n(w-\varphi)}{2}\right] d S .
\end{aligned}
$$


Here $f^{k}$ is again a vector field that points outward on $B$, and $c$ is determined by both $a^{i j}$ and $f^{k}$ in the inequality (3.8).

The right-hand side of (3.29) can be computed explicitly. If $\mathfrak{A}(\varphi)$ approximates $\mathfrak{A}(w)$ in mean square, and if $\varphi$ and its tangential gradient on $B$ approximate $w$ and its tangential gradient, then the bound for $A[w-\varphi]$ is small, and (3.21) gives close upper and lower bounds for $A[w]$. From the computational point of view, it is easier to make a quadratic form small. Consequently, we replace (3.29) by the slightly worse estimate

$$
\begin{gathered}
A[w-\varphi] \leqq \frac{1}{a_{0} \lambda_{1}} \iint_{D}[\mathfrak{A}(w)-\mathfrak{A}(\varphi)]^{2} d V \\
+\oint_{B} \frac{1}{f^{k} n^{k}}\left\{\beta n^{-1}\left[\left(f^{k} n_{k}\right)^{2}+\left(a_{i j} f^{i} t^{\jmath}\right)^{2}\right]\left[\frac{\partial}{\partial t}(w-\varphi)\right]^{2}\right. \\
\left.-(\beta c+1) a_{i j} f^{i} t^{\jmath}(w-\varphi) \frac{\partial}{\partial t}(w-\varphi)+\left(\frac{\beta c^{2}}{4}+\frac{c}{2}+\frac{1}{4 \beta}\right) n(w-\varphi)^{2}\right\} d S .
\end{gathered}
$$

Here $\beta$ is an arbitrary positive constant. The optimum value of $\beta$ yields (3.29) again. If, however, we fix $\beta$, the right-hand side of (3.30) is a quadratic form in $(w-\varphi)$. Thus we can take for $\varphi$ an undetermined linear combination

$$
\varphi=\sum_{i=1}^{k} b_{i} \varphi_{i}
$$

where the $\varphi_{i}$ are arbitrary functions. The right-hand side of (3.30) is then a non-homogeneous quadratic form in the coefficients $b_{i}$, so that minimization with respect to the $b_{i}$ reduces to inverting a $k$ by $k$ matrix. This is just the well-known Rayleigh-Ritz method.

Improved bounds can be obtained when either the differential equation or the boundary condition is homogeneous. For example, let $w$ satisfy

$$
\mathfrak{A}(w)=0 .
$$

Then if we choose $\varphi$ to satisfy the same equation

$$
\mathfrak{A}(\varphi)=0
$$

we find the identity

$$
A[w]=2 \oint_{B} w \frac{\partial \varphi}{\partial \nu} d S-A[\varphi]-A[w-\varphi] .
$$

All but the last term on the right can be computed explicitly. The fact that $A[w-\varphi]$ is non-negative gives a lower bound for $A[w]$ (this is just 
Thomson's principle). The inequality (3.29) or (3.30) yields an upper bound. The error term is homogeneous of degree two (rather than one as in (3.21)) in the boundary values of $w-\varphi$ and $\partial / \partial t(w-\varphi)$.

A similar situation occurs if we take $\varphi$ to satisfy

$$
\varphi=w \text { on } B \text {. }
$$

In this case we have the identity

$$
A[w]=A[\varphi]-A[w-\varphi] .
$$

The upper bound is just Dirichlet's principle, while a lower bound is obtained with the aid of (3.29) or (3.30). Again, the error term is of degree two in $w-\varphi$. Such a result also occurs when

$$
w=0 \text { on } B,
$$

and $\varphi$ is chosen to satisfy either the differential equation or the boundary condition.

In the special case in which $\mathfrak{A}$ is the Laplace operator a number of methods for obtaining bounds in harmonic problems have been proposed (see in particular Diaz [2], and the papers referred to in the bibliographies of [2] and [10]).

4. Pointwise bounds in Dirichlet's problems. We now give bounds for the value at an interior point $p \sim\left(x_{p}^{1}, \cdots, x_{p}^{N}\right)$ of the solution $w$ of a Dirichlet problem. As in section (3c), we suppose that $\mathfrak{A}(w)$ is given in $D$ and the value of $w$ itself is given on the boundary $B$.

We again consider the arbitrary function $\varphi$ which is chosen so that $\mathfrak{H}(\varphi)$ is close to $\mathfrak{A}(w)$ and the values of $\varphi$ on $B$ are close to those of $w$. We shall approximate the value of $w$ at $p$ not by $\varphi(p)$, but by a functional depending only on $\varphi$ and the given data for $w$.

For this purpose we first introduce the function $\Gamma_{p}(x)$ having the following properties ${ }^{1}$ :

(a) At all points of $D$ except $p, \Gamma_{p}(x)$ is twice continuously differentiable, so that $\mathfrak{U}\left(\Gamma_{p}\right)$ is continuous except at $p$;

(b) $\left[\sum_{i=1}^{N}\left(x^{i}-x_{p}^{i}\right)\right]^{\frac{N-1}{2}}\left[\mathfrak{A}\left(\Gamma_{p}\right)\right]^{2}$ is integrable over $D$;

(c) $\Gamma_{p}$ is singular at $P$ in such a manner that if $S_{\rho}$ is a sphere of radius $\rho$ centered at $P, \lim _{\rho \rightarrow 0} \oint_{S_{\rho}} \frac{\partial \Gamma_{p}}{\partial \nu} d S=1$

where $\partial / \partial \nu$ is the outward conormal derivative.

1 As pointed out by J. B. Diaz the function $\Gamma_{p}$ is the so called "parametrix" introduced by E. E. Levi, (Rend. Circ Mat. Palermo 24 (1907), 275-317). 
If $\mathfrak{A}\left(\Gamma_{p}\right)=0, \Gamma_{p}$ is just a fundamental solution. However, such a function may be quite difficult to construct. On the other hand, one can always take

$\Gamma_{p}(x)= \begin{cases}\frac{1}{4 \pi}\left|a\left(x_{p}\right)\right|^{1 / 2} \log \left\{a_{i j}\left(x_{p}\right)\left(x^{i}-x_{p}^{i}\right)\left(x^{j}-x_{p}^{j}\right)\right\} & \text { for } N=2 \\ \frac{1}{(N-2) \omega_{N}}\left|a\left(x_{p}\right)\right|^{1 / 2}\left\{a_{i j}\left(x_{p}\right)\left(x^{i}-x_{p}^{i}\right)\left(x^{j}-x_{p}^{j}\right)\right\}^{-\left(\frac{N-2}{2}\right)} & \text { for } N>2\end{cases}$

where $a$ is the determinant of $a_{i j}$, and $\omega_{N}$ is the surface of the $N$ dimensional unit sphere.

By means of Green's identity (3.27) we find the representation

$$
\begin{aligned}
w(p)-\varphi(p)= & \iint_{D}\left\{\Gamma_{p}[\mathfrak{Y}(w)-\mathfrak{U}(\varphi)]-(w-\varphi) \mathfrak{Y}\left(\Gamma_{p}\right)\right\} d V \\
& +\oint_{B}\left\{(w-\varphi) \frac{\partial \Gamma_{p}}{\partial \nu}-\Gamma_{p} \frac{\partial}{\partial \nu}(w-\varphi)\right\} d S .
\end{aligned}
$$

In order to bound the last term on the right, we again write $w-\varphi=$ $u+v$, where $u=0$ on $B$ and $\mathfrak{A}(v)=0$. Then

$$
\begin{array}{r}
\oint_{B} \Gamma_{p} \partial_{\partial \nu}^{\partial}(w-\varphi) d S+\oint_{B} \frac{\Gamma_{p}}{f^{k} n_{k}}\left[a_{i j} f^{i} t^{\jmath} \frac{\partial}{\partial t}(w-\varphi)-\frac{c n}{2}(w-\varphi)\right] d S \\
=\oint_{B} \Gamma_{p}\left[\frac{\partial v}{\partial \nu}+\frac{\left(a_{i j} f^{i} t^{j} \frac{\partial v}{\partial t}-\frac{c n v}{2}\right)}{f^{k} n_{k}}\right] d S+\oint_{B} I_{p} \frac{\partial u}{\partial \nu} d S,
\end{array}
$$

where $f^{k}$ and $t^{k}$ have the same significance as in section $3 \mathrm{~b}$. Since $u=0$ on $B, v=w-\varphi$ and $\partial v / \partial t=\partial / \partial \iota(w-\varphi)$ on $B$, the second term on the left is known. We subtract this term from both sides of (4.3), transpose the second and third terms (which are known), and apply Schwarz's inequality together with (3.12) and (3.17) to find

$$
\begin{aligned}
& |w(p)-\chi(p)| \leqq\left[\oint_{B} \frac{n \Gamma_{p}^{2}}{f^{k} n_{k}} d S\right]^{1 / 2}\left\{\left[\oint _ { B } \frac { n ^ { - 1 } } { f ^ { k } n _ { k } } \left\{\left(f^{k} n_{k} \frac{\partial}{\partial t}(w-\varphi)\right)^{2}\right.\right.\right. \\
& \left.\left.\left.+\left(a_{i j} f^{i} t^{j} \frac{\partial}{\partial t}(w-\varphi)-\frac{c n(w-\varphi)}{2}\right)^{2}\right\} d S\right]^{1 / 2}+\left(K \iint_{D}[\mathfrak{U}(w-\varphi)]^{2} d V\right)^{1 / 2}\right\} \\
& +\left|\iint_{D}(w-\varphi) \mathfrak{A}\left(\Gamma_{p}\right) d V\right| .
\end{aligned}
$$

Here we have written 


$$
\begin{gathered}
\chi(p)=\varphi(p)+\iint_{D} \Gamma_{p}[\mathfrak{U}(w)-\mathfrak{U}(\varphi)] d V \\
+\oint_{B}\left\{(w-\varphi) \frac{\partial \Gamma_{p}}{\partial \nu}+\frac{\Gamma_{p}}{f^{k} n_{k}}\left[a_{i j} f^{i} t^{j} \frac{\partial}{\partial t}(w-\varphi)-\frac{c n(w-\varphi)}{2}\right]\right\} d S .
\end{gathered}
$$

It is only necessary to bound the last term on the right of (4.5). We first consider the three-dimensional case, $N=3$. Let

$$
r^{2}=\sum_{i=2}^{3}\left(x^{i}-x_{p}^{i}\right)^{2} .
$$

By the divergence theorem we have for any function $\psi(x)$

$$
2 \iint_{D} \frac{\left(x^{i}-x_{p}^{i}\right)}{r^{2}} \psi \psi_{, i} d V=\oint_{B} \frac{\left(x^{i}-x_{p}^{i}\right) n_{i} \psi^{2}}{r^{2}} d S-\iint_{D} \frac{\psi^{2}}{r^{2}} d V
$$

Applying Schwarz's inequality and completing the square yields

$$
\begin{aligned}
\left\{\iint_{D} \frac{\psi^{2}}{r^{2}} d V\right\}^{1 / 2} & \leqq\left\{\iint_{D}|\operatorname{grad} \psi|^{2} d V\right\}^{1 / 2} \\
& +\left\{\iint_{D}|\operatorname{grad} \psi|^{2} d V+\oint_{B} \frac{\left(x^{i}-x_{p}^{i}\right)}{r^{2}} n_{\iota} \psi^{2} d S\right\}^{1 / 2} .
\end{aligned}
$$

It follows from (2.2) that

$$
\iint_{D}|\operatorname{grad} \psi|^{2} d V \leqq \frac{1}{a_{0}} A[\psi] .
$$

If we let $\psi=w-\varphi$, and use the bound (3.30) for $A[w-\varphi]$, (4.9) yields a bound for $\iint_{D} r^{-2}(w-\varphi)^{2} d V$. Thus, we can apply Schwarz's inequality to the last term of (4.5) to obtain

$$
\left|\iint_{D}(w-\varphi) \mathfrak{A}\left(\Gamma_{p}\right) d V\right| \leqq\left\{\iint_{D} r^{-2}(w-\varphi)^{2} d V\right\}^{1 / 2}\left\{\iint_{D} r^{2}\left[\mathfrak{A}\left(\Gamma_{p}\right)\right]^{2} d V\right\}^{1 / 2}
$$

The second factor on the right converges because of condition (4.1b), and is explicitly known. The first factor is explicitly bounded by (4.9), (4.10) and (3.30). Thus, all the terms on the right of (4.5) are known explicitly. In fact, this right-hand side is a linear combination of square roots of quadratic forms in $(w-\varphi)$ and $\partial / \partial t(w-\varphi)$ on $B$, and $\mathscr{A}(w)-\mathfrak{A}(\varphi)$ in $D$. By means of the inequality

$$
\text { (4.12) }(a+b+c)^{2} \leqq\left(1+\alpha+\frac{1}{\beta}\right) a^{2}+\left(1+\beta+\frac{1}{\gamma}\right) b^{2}+\left(1+\gamma+\frac{1}{\alpha}\right) c^{2}
$$

where $\alpha, \beta$ and $\gamma$ are any positive numbers, one can bound the right-hand side of (4.5) with the square root of a single explicitly known quadratic functional. This can then be made arbitrarily small by the RaleighRitz method. Thus, the bounds for $w(P)$ can be made arbitrarily close. 
For $N=2$, we replace the identity (4.8) by

$$
2 \iint_{D} \frac{\left(x^{i}-x_{p}^{i}\right)}{r} \psi \psi_{, i} d V=\oint_{B} \frac{\left(x^{i}-x_{p}^{i}\right)}{r} n_{i} \psi^{2} d S-\iint_{D} \frac{\psi^{2}}{r} d V
$$

to derive

$$
\begin{aligned}
\left\{\iint_{D} \frac{\psi^{2}}{r} d V\right\}^{1 / 2} \leqq & \left\{\iint_{D} r|\operatorname{grad} \psi|^{2} d V\right\}^{1 / 2} \\
& +\left\{\iint_{D} r|\operatorname{grad} \psi|^{2} d V+\oint_{B} \frac{\left(x^{i}-x^{i}\right)}{r} n_{i} \psi^{2} d S\right\}^{1 / 2} .
\end{aligned}
$$

Now

$$
\iint_{D} r|\operatorname{grad} \varphi|^{2} d V \leqq \frac{r_{\max }}{a_{0}} A[\varphi],
$$

where $r_{\max }$ is the maximum distance of a point on $B$ from $p$.

By Schwarz's inequality

$$
\left|\iint_{D}(w-\varphi) \mathfrak{A}\left(\Gamma_{p}\right) d V\right| \leqq\left\{\iint_{D} \frac{(w-\varphi)^{2}}{r} d V\right\}^{1 / 2}\left\{\iint_{D} r\left[\mathfrak{M}(\Gamma)^{2} d V\right\}^{1 / 2}\right.
$$

and the right-hand side is again explicitly bounded.

For $N>3$, we let

$$
\rho^{2}=a_{i j}\left(x_{p}\right)\left(x^{i}-x_{p}^{i}\right)\left(x^{j}-x_{p}^{j}\right)
$$

and put

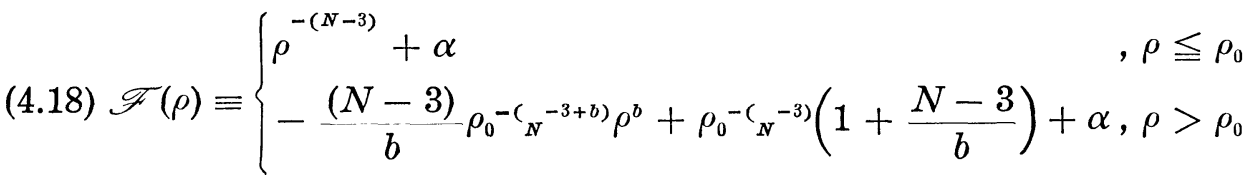
where $\rho_{0}, b$, and $\alpha$ are constants to be specified later. $\mathscr{F}(\rho)$ is continuously differentiable for $\rho>0$ and has continuous second derivatives except for $\rho=\rho_{0}$ and $\rho=0$.

For small $\rho$ we have

$$
\mathfrak{A}(\mathscr{F})=-\frac{(N-3)}{\rho_{N}^{-1}}+O\left(\rho^{-(N-2)}\right) .
$$

Thus, $\mathfrak{A}(\mathscr{F})$ is negative for sufficiently small $\rho$, and we choose $\rho_{0}$ so that $\mathfrak{A}(\mathscr{F})<0$ for $\rho \leqq \rho_{0}$. For $\rho>\rho_{0}$

$$
\begin{aligned}
\mathfrak{A}(\mathscr{F})= & -(N-3) \rho_{0}^{-(N-3+b)}\left\{\left[a^{i j}(x) a_{i j}\left(x_{p}\right)+a_{, j}^{i j}(x) a_{i k}\left(x_{p}\right)\left(x^{k}-x_{p}^{k}\right)\right] \rho^{b-2}\right. \\
& \left.+(b-2) a^{i j}(x) a_{i k}\left(x_{p}\right) a_{j l}\left(x_{p}\right)\left(x^{k}-x_{p}^{k}\right)\left(x^{l}-x_{p}^{l}\right) \rho^{b-4}\right\}
\end{aligned}
$$

Noting that the second term in the braces is bounded below for $b \geqq 2$ by $(b-2) a_{0} a_{1}^{-1} \rho^{(b-2)}$, we can make $\mathfrak{A}(\mathscr{F})$ negative throughout $D$ by choosing $b$ sufficiently large, i.e., 


$$
b-2 \geqq \max _{\rho \geqq \rho_{0}}\left\{O, \frac{a^{i j}(x) a_{j}\left(x_{p}\right)+a_{, j}^{i j}(x) a_{i k}\left(x_{p}\right)\left(x^{k}-x_{\rho}^{k}\right)}{a^{i j}(x) a_{, k}\left(x_{p}\right) a_{j l}\left(x_{p}\right)\left(x^{k}-x_{p}^{k}\right)\left(x^{l}-x_{p}^{l}\right)} \rho^{2}\right\} .
$$

Finally, $\alpha$ is chosen so large that $\mathscr{F}$ is positive in $D$. By Green's identity (3.27)

$$
\begin{aligned}
& \iint_{D} \mathscr{F} a^{i j}(w-\varphi)_{, i}(w-\varphi)_{, j} d V+\iiint_{D}\left[-\frac{1}{2} \mathfrak{A}(\mathscr{F})(w-\varphi)^{2}\right] d V \\
& \quad=\oint_{B}\left[\mathscr{F}(w-\varphi) \frac{\partial}{\partial \nu}(w-\varphi)-\frac{1}{2}(w-\varphi)^{2} \frac{\partial \mathscr{F}}{\partial \nu}\right] d S \\
& \quad-\iint_{D} \mathscr{F}(w-\varphi) \mathfrak{A}(w-\varphi) d V .
\end{aligned}
$$

By the construction of $\mathscr{F}$ both terms on the left are positive. Dropping the first of them and applying Schwarz's inequality and the inequalities (3.12) and (3.17) one obtains

$$
\begin{aligned}
& \left\{\iint_{D}-\mathfrak{A}(\mathscr{F})(w-\varphi)^{2} d V\right\}^{1 / 2} \leqq\left\{\iint_{D} \frac{\mathscr{F}^{2}[\mathfrak{A}(w)-\mathfrak{A}(\varphi)]^{2}}{-\mathfrak{A}(\mathscr{F})} d V\right\}^{1 / 2} \\
& +\left\{\iint_{D} \frac{\mathscr{F}^{2}[\mathfrak{H}(w)-\mathfrak{A}(\varphi)]^{2}}{-\mathfrak{U}(\mathscr{F})} d V-\oint_{B}\left[(w-\varphi)^{2} \frac{\partial \mathscr{F}}{\partial \nu}\right.\right. \\
& \left.+2 \frac{\mathscr{F}(w-\varphi)}{f^{k} n_{k}}\left\{a_{i j} f^{i} t^{j} \frac{\partial}{\partial t}(w-\varphi)-\frac{c n}{2}(w-\varphi)\right\}\right] d S \\
& +2\left(\oint_{B} \frac{n \mathscr{F}^{2}(w-\varphi)^{2}}{f^{k} n_{k}} d S\right)^{1 / 2}
\end{aligned}
$$

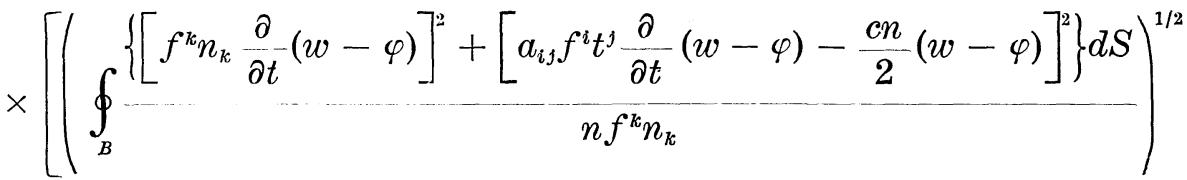

$$
\begin{aligned}
& \left.\left.+\left(K \iint_{D}[\mathfrak{A}(w)-\mathfrak{A}(\varphi)]^{2} d V\right)^{1 / 2}\right]\right\}^{1 / 2} .
\end{aligned}
$$

The last term of (4.5) is now bounded by the Schwarz's inequality

$$
\left|\iint_{D}(w-\varphi) \mathfrak{A}\left(\Gamma_{\rho}\right) d V\right| \leqq\left\{\iint_{D}-\mathfrak{U}(\mathscr{F})(w-\varphi)^{2} d V\right\}^{1 / 2}\left\{\iint_{D} \frac{\left[\mathfrak{A}\left(\Gamma_{\mathrm{\rho}}\right)\right]^{2}}{-\mathfrak{A}(\mathscr{F})} d V\right\}^{1 / 2}
$$

The last factor converges because of (4.19) and condition (4.1b), and can be computed explicitly. The first factor is bounded by (4.23). The righthand side of (4.5) may thus again be bounded by the square root of a quadratic form in $w-\varphi$ and $\partial / \partial t(w-\varphi)$ on $B$ and $\mathfrak{A}(w-\varphi)$ in $D$, which can be made arbitrarily small by the Rayleigh-Ritz method. Thus arbitrarily close upper and lower bounds for $w(P)$ are obtained in any number of dimensions. 
To find a given derivative of $w$ at $p$, it is only necessary to differentiate the representation (4.3). We can then proceed exactly as before, providing only that the given derivatives of $\varphi, \Gamma_{p}(x)$, and $\mathfrak{A}\left(\Gamma_{p}\right)$ with respect to the coordinates $x_{p}^{i}$ exist and are continuous for $x_{p} \neq x$, and the derivative of $\Gamma_{p}(x)$ again satisfies conditions (4.1a) and (4.1b). In general for variable $a^{i j}$, the derivatives of the functions (4.2) will violate condition (4.1b). If the $a^{i j}$ are twice continuously differentiable, it is easily verified that in two dimensions the function

$$
\Gamma_{p}(x)=\frac{1}{4 \pi}\left|a(x) a\left(x_{p}\right)\right|^{1 / 4} \log \left\{\left[a_{i j}(x)+a_{i j}\left(x_{p}\right)\right]\left(x^{i}-x_{p}^{i}\right)\left(x^{j}-x_{p}^{j}\right)\right\}
$$

satisfies (4.1a, b, and $c$ ) and its first derivatives satisfy (4.1a and b). In $N$ dimensions with $N \geqq 3$, one uses the function

(4.26) $\Gamma_{p}(x)$

$$
=\frac{2^{\frac{N-2}{2}}}{(N-2) \omega_{N}}\left|a(x) a\left(x_{p}\right)\right|^{1 / 4}\left\{\left[a_{i j}(x)+a_{i j}\left(x_{p}\right)\right]\left(x^{i}-x_{p}^{i}\right)\left(x^{j}-x_{p}^{j}\right)\right\}^{-\frac{(N-2)}{2}} .
$$

Thus one can always estimate the derivatives of the solution of Dirichlet's problem with arbitrary accuracy.

It is clear that great simplifications in the above results occur if $\Gamma_{p}(x)$ is a fundamental solution, that is, if $\mathfrak{A}\left(I_{p}\right)=0$, so that the last term in (4.5) is absent.

5. The exterior mixed problem. Let $u$ be an arbitrary twice differentiable function defined in the exterior $D$ of a closed surface $B$ in $N$ - space $(N \geqq 3)$. We assume that at infinity $u$ tends to zero in such a way that the integrals of $r^{-2} u^{2}$ and $r^{2}[\mathfrak{U}(u)]^{2}$ exist, where $r$ is the distance from some origin inside $B$. We further assume that $\int_{S_{R}} u \partial u / \partial \nu d S$ approaches zero as $R \rightarrow \infty$, where $S_{R}$ is the sphere of radius $R$ centered at the origin. (These conditions imply the existence of $A[u]$ ).

Finally, we let the boundary $B$ consist of two parts $B_{1}$ and $B_{2}$ and suppose that $u$ vanishes on $B_{1}$. A non-negative function $h$ is given on $B_{2}$.

Let $g^{k}(x), k=1,2,3, \cdots, N$ be a continuous piecewise differentiable vector field defined in $D$ and satisfying

$$
G \equiv g_{, i}^{i}-a_{i j} g^{i} g^{j}>0 \quad \text { in } D
$$

and

$$
\tilde{G} \equiv-g^{k} n_{k}+h>0 \quad \text { on } B_{2}
$$


where $n_{k}$ is the unit normal on $B$ pointing outward from $D$ (into the interior of $B$ ). Furthermore, we assume that

$$
g^{k}=O\left(r^{-1}\right) \text { as } r \rightarrow \infty
$$

and

$$
g_{, k}^{k}=O\left(r^{-2}\right) \text { as } r \rightarrow \infty
$$

If $B_{2}$ is star-shaped with respect to the origin, one can take

$$
g^{k}=\alpha x^{k} r^{-2} \quad O<\alpha<(N-2) / a_{1},
$$

where $a_{1}$ is the constant defined in (2.2). Otherwise, one can take this function outside a sphere containing $B$, and continue it in such a way that $g^{k}$ satisfies (5.1) and (5.2).

From the divergence theorem it follows that

$$
\iint_{D} g{ }_{, k}^{k} u^{2} d V-\int_{B_{2}} g^{k} n_{k} u^{2} d S=-2 \iint_{D} g^{k} u u_{, k} d V .
$$

Since a scalar product is less than half the sum of the squares of the norms,

$$
-2 g^{k} u u_{, k} \leqq a_{\imath \jmath} g^{i} g^{j} u^{2}+a^{i j} u_{, i} u,
$$

Combining (5.6) and (5.7) we find that

$$
\iint_{D} G u^{2} d V+\int_{B_{2}} \tilde{G} u^{2} d S \leqq A[u]+\int_{B_{2}} h u^{2} d S .
$$

Now

$$
\begin{aligned}
& \left\{A[u]+\int_{B_{2}} h u^{2} d S\right\}^{2}=\left\{-\iint_{D} u \mathscr{A}(u) d V+\int_{B: 2} u\left(\frac{\partial u}{\partial \nu}+h u\right) d S\right\}^{2} \\
& \quad \leqq\left\{\iint_{D} G u^{2} d V+\int_{B_{2}} \tilde{G} u^{2} d S\right\}\left\{\iint_{D}^{[\mathfrak{U}(u)]^{2}} \frac{\left(\frac{\partial u}{\partial \nu}+h u\right)^{2}}{G} d V+\int_{B_{2}} \frac{\tilde{G}}{\tilde{G}} d S\right\} .
\end{aligned}
$$

Squaring both sides of (5.8), inserting (5.9), and dividing by the common factor yields

$$
\iint_{D} G u^{2} d V+\int_{B_{2}} \tilde{G} u^{2} d V \leqq \iint_{D} \frac{[\mathfrak{A}(u)]^{2}}{G} d V+\int_{B_{2}} \frac{\left(\frac{\partial u}{\partial \nu}+h u\right)^{2}}{\widetilde{G}} d S .
$$

On the other hand, inserting (5.10) into (5.9) yields

$$
A[u]+\int_{B_{2}} h u^{2} d S \leqq \iint_{D} \frac{[\mathfrak{A}(u)]^{2}}{G} d V+\int_{B_{2}} \frac{\left(\frac{\partial u}{\partial \nu}+h u\right)^{2}}{\tilde{G}} d S .
$$


Thus, when $g^{k}$ satisfies (5.1) and (5.2), we have bounds for the integral of $u^{2}$ in $D$ and on $B_{2}$, as well as for $A[u]+\int_{B_{2}} h u^{2} d S$ in terms of a quadratic functional in $\mathfrak{A}(u)$ and $\partial u / \partial \nu+h u$.

Let us now consider the mixed boundary value problem for a function $w(x)$. That is, $\mathfrak{A}(w)$ is given in $D, w$ is given on $B_{1}$, and $\partial w / \partial \nu+h w$ is given on $B_{2}$. We assume that $w=O\left(r^{-(}{ }_{N}{ }^{-2)}\right)$ as $r \rightarrow \infty$. Let $\varphi$ be an arbitrary function of order $r^{-(}{ }_{N}{ }^{-2)}$ at infinity which takes the given values of $w$ on $B_{1}$. Let $u=w-\varphi$. We suppose $\mathfrak{A}(\varphi)$ to approximate $\mathfrak{A}(w)$ sufficiently well that $\iint_{D}[\mathfrak{T}(u)]^{2} d V$ converges. Then $A[w]+\int_{B_{2}} h w^{2} d S$ is estimated by

$$
\left|\left\{A[w]+\int_{B_{2}} h w^{2} d S\right\}^{1 / 2}-\left\{A[\varphi]+\int_{B_{2}} h \varphi^{2} d S\right\}^{1 / 2}\right| \leqq\left\{A[u]+\int_{B_{2}} h u^{2} d S\right\}^{1 / 2} \cdot
$$

Thus, the upper and lower bounds may be made close by mimimizing the right-hand side of (5.11) with respect to a family of admissible functions $\varphi$ (Rayleigh-Ritz technique).

Bounds at an interior point $p$ follow as in $\S 4$. We write down the representation (4.3), transpose known terms, and apply Schwarz's inequality and (5.10) to find

$$
\begin{aligned}
& \left\{w(p)-\varphi(p)-\iint_{D} \Gamma_{p}[\mathfrak{A}(w)-\mathfrak{A}(\varphi)] d V\right. \\
& \left.\quad-\int_{B_{2}} \Gamma_{p}\left[\frac{\partial}{\partial \nu}(w-\varphi)+h(w-\varphi)\right] d S\right\}^{2} \\
& \quad \leqq\left\{\iint_{D} \frac{\left[\mathfrak{A}\left(\Gamma_{p}\right)\right]^{2}}{G} d V+\int_{B_{2}} \frac{\left(\frac{\partial \Gamma_{p}}{\partial \nu}+h \Gamma_{p}\right)^{2}}{G} d S\right\} \\
& \quad \times\left\{\iint_{D} \frac{[\mathfrak{A}(w-\varphi)]^{2}}{G} d V+\int_{B_{2}} \frac{\left[\frac{\partial}{\partial \nu}(w-\varphi)+h(w-\varphi)\right]^{2}}{\tilde{G}} d S\right\} .
\end{aligned}
$$

Here $\Gamma_{p}$ is a function having the properties (4.1) vanishing on $B_{1}$, and satisfying the conditions that $\Gamma_{p}=O\left(r^{-}{ }_{N}{ }^{-2)}\right)$ and $\mathfrak{A}\left(\Gamma_{p}\right)=O\left(r^{-}{ }_{N}\right)$ as $r \rightarrow \infty$. We note that everything on the left of (5.13) except $w(p)$ is known, so that upper and lower bounds for $w(p)$ are obtained. They are close if the right-hand side is small. This depends on minimizing a quadratic functional (the second factor on the right of (5.13)) with respect to admissible functions $\varphi$.

We note that the first factor on the right of (5.13) is the same quadratic form in $\Gamma_{p}$. Since it is easy to find a whole family of admissible $\Gamma_{p}$ (one $\Gamma_{p}$ plus any sufficiently regular function will do), one can 
minimize the first factor also. This may result in considerable improvement of the bounds.

The same procedure may be applied to the interior mixed boundary value problem. However, the construction of the vector field $g^{k}$ satisfying conditions (5.1) and (5.2) becomes more difficult. (Conditions (5.3) and (5.4) are clearly unnecessary here). If $B_{2} \equiv B$ and $h$ vanishes identically (Neumann problem), it is an immediate consequence of the divergence theorem that no such vector field $g^{k}$ exists.

On the other hand, if $h$ is everywhere positive and $B_{2} \equiv B$ is star shaped, the vector $\alpha x^{k}$ with $\alpha$ sufficiently small satisfies (5.1) and (5.2).

6. More general operators. All the results which have been presented in the preceding sections for the special operator $\mathfrak{A}(u)$ can be extended to elliptic second order operators of the form

$$
\mathscr{B}(u)=\left(a^{i j}(x) u_{, i}\right)_{, j}-b^{i}(x) u_{, i}-q(x) u \text {. }
$$

The coefficients $a^{i j}$ and $b^{i}$ are assumed piecewise continuously differentiable in $D$, while $q$ is assumed bounded. We must, of course, exclude the occurrence of non-positive eigenvalues of this operator. To this effect, we assume

$$
q(x) \geqq 0
$$

and the existence of two positive constants $b_{0}$ and $b_{1}$ such that for all $x \in D$ and all real numbers $\left(\xi_{0}, \xi_{1}, \cdots, \xi_{N}\right)$.

(6.3) $\quad b_{0}\left[\sum_{i=1}^{N} \xi_{i}^{2}+q(x) \xi_{0}^{2}\right] \leqq a^{i j} \xi_{i} \xi_{j}+b^{i} \xi_{0} \xi_{i}+q(x) \xi_{0}^{2} \leqq b_{1}\left[\sum_{i=1}^{N} \xi_{i}^{2}+q(x) \xi_{0}^{2}\right]$.

It is clear that the inequalities (3.5), (3.6), and (3.7) remain valid when the quadratic form $A[u]$ is replaced by

$$
B[u]=\iint_{D}\left[a^{i j} u_{, i} u, b_{, j}^{i} u u_{, i}+q u^{2}\right] d V,
$$

$\mathfrak{A}(u)$ by $\mathscr{B}(u)$, and the constant $a_{0}$ by $b_{0}$ (Actually, $\lambda_{1}$ may be replaced by $\left.\lambda_{1}+\min q\right)$.

In the identity (2.14) one uses the fact that

$$
\mathfrak{A}(u)=\mathscr{B}(u)+b^{i} u_{, i}+q u \text {. }
$$

Then the right-hand side becomes the integral of $-2 f^{i} u_{, i} \mathscr{B}(u)$ plus a quadratic form in $u$ and its first derivatives. Because of (6.3), the latter part is bounded by

$$
-\bar{c}\left[a^{i j} u_{, i} u_{, j}+b^{i} u u_{, i}+q u^{2}\right],
$$

where $\bar{c}$ is a constant. This replaces the inequality (3.8). One can now 
follow the procedure of section (3a) to arrive at inequalities of the form (3.12) and (3.14) for a function $u$ vanishing on the boundary with $\mathfrak{2}(u)$ replaced by $\mathscr{B}(u)$. It is only necessary to replace $a_{0}$ by $b_{0}$ and $\bar{c}$ by $c$ in the definition (3.13) of $K$.

In a similar fashion one finds the inequality (3.17) with $c$ replaced by $\bar{c}$, and the inequality (3.19) with $A[v]$ replaced by $B[v]$ and $c$ by $\bar{c}$ for a function $v$ satisfying $\mathscr{P}(v)=0$.

Thus, the quadratic from $B[w]$ of the solution of Dirichlet's problem can again be estimated with arbitrary accuracy. The triangle inequality

$$
\{B[\varphi]\}^{1 / 2}-\{B[w-\varphi]\}^{1 / 2} \leqq\{B[w]\}^{1 / 2} \leqq\{B[\varphi]\}^{1 / 2}+\{B[w-\varphi]\}^{1 / 2}
$$

holds, and $B[w-\varphi]$ is bounded by the inequality (3.29) with $A[w-\varphi]$ replaced by $B[w-\varphi], \mathscr{A}(w-\varphi)$ by $\mathscr{B}(w-\varphi), a_{0}$ by $b_{0}$, and $c$ by $\bar{c}$.

In the pointwise approximation of the solution $w$ of Dirichlet's problem we replace the representation (4.3) by

$$
\begin{aligned}
w(p)-\varphi(p)= & \iint_{D}\left\{\Gamma_{p} \mathscr{B}(w-\varphi)-(w-\varphi) \mathscr{P}^{*}\left(\Gamma_{p}\right)\right\} d V \\
& +\oint_{B}\left\{(w-\varphi)\left(\frac{\partial \Gamma_{p}}{\partial \nu}+b^{i} n_{i} \Gamma_{p}\right)-\Gamma_{p} \frac{\partial}{\partial \nu}(w-\varphi)\right\} d S .
\end{aligned}
$$

Here $\mathscr{B}^{*}$ is the adjoint operator

$$
\mathscr{B}^{*}(u)=\left(a^{i j} u,{ }_{, j}+\left(b^{i} u\right)_{, i}-q u\right.
$$

and $\Gamma_{p}$ is again a singular function satisfying the conditions (4.1) but with $\mathscr{A}\left(\Gamma_{p}\right)$ replaced by $\mathscr{B}^{*}\left(\Gamma_{p}\right)$. (The function (4.2) still satisfies these conditions). The pointwise bounds are now found exactly as in $\S 4$.

Section 5 is also easily extended to operators of the form $\mathscr{B}(u)$. It is only necessary to recall that $B[u] \geqq b_{0} A[u] / a_{1}$ and that Green's Theorem

$$
B[u]=-\iint_{D} u \mathscr{B}(u) d V+\oint_{B} u \frac{\partial u}{\partial \nu} d S
$$

holds, in order to obtain inequalities like (5.8) through (5.11) with $A[u]$ replaced by $B[u]$ and $\mathscr{A}(u)$ by $\mathscr{S}(u)$. These, together with the representation (6.8), yield the pointwise approximation.

7. Second order systems. The results are just as easily extended to a large class of elliptic systems of second order equations". We simply substitute an $M$-vector for the unknown function $u$ and $M \times M$ matrices for the coefficients in the definition (6.1) of $\mathscr{S}(u)$. Then we have

2 The extension to systems was suggested to the authors by A. Douglis. 


$$
\mathscr{B}_{\alpha}(u)=\left(a_{\alpha \beta}^{i j} u_{, j}^{\beta}\right)_{, i}-b_{\alpha \beta}^{i} u_{, i}^{\beta}-q_{\alpha \beta} u^{\beta}, \quad \alpha, \beta=1, \cdots, M .
$$

The coefficients $a_{\alpha \beta}^{i j}$ and $b_{\alpha \beta}^{i}$ are again piecewise differentiable and $q_{\alpha \beta}$ is assumed bounded. The $a_{\alpha \beta}^{i j}$ are such that $a_{\alpha \beta}^{i j}=a_{\alpha \beta}^{j i}$. We replace (6.2) by

$$
q_{\alpha \beta} \xi^{a} \xi^{\beta} \geqq 0
$$

for any $M$-vector $\xi^{a}$, and

$$
\begin{aligned}
b_{0}\left[\sum_{i=1}^{N} \sum_{\alpha=1}^{M}\left(\xi_{i}^{\alpha}\right)^{2}+q_{\alpha \beta} \xi^{a} \xi^{\beta}\right] & \leqq a_{\alpha \beta}^{i j} \xi_{i}^{\alpha} \xi_{j}^{\beta}+b_{\alpha \beta}^{i} \xi_{i}^{\alpha} \xi_{0}^{\beta}+q_{\alpha \beta} \xi_{0}^{\alpha} \xi_{0}^{\beta} \\
& \leqq b_{1}\left[\sum_{i=1}^{N} \sum_{\alpha=1}^{M}\left(\xi_{i}^{\alpha}\right)^{2}+q_{\alpha \beta} \xi_{0}^{\alpha} \xi_{0}^{\beta}\right],
\end{aligned}
$$

for two positive constants $b_{0}$, and $b_{1}$, any set of $N+1 M$-vectors $\left(\xi_{0}^{\alpha}, \xi_{1}^{\alpha}, \cdots, \xi_{N}^{\alpha}\right)$ and any $x \in D$.

The equations of the classical theory of elasticity [13] satisfy these conditions. Methods for obtaining bounds for this particular system of equations have been given by Diaz and Greenberg [3] and Synge [14]. Their method differs considerably from the one proposed here. The analogue of the conormal derivative is the $M$-vector.

$$
\left(\frac{\partial u}{\partial \nu}\right)_{\alpha}=a_{\alpha \beta}^{i j} u_{, j}^{\beta} n_{i} \text {. }
$$

In terms of this vector, we have the Green's theorem

$$
\iint_{D} u^{a} \mathscr{A}_{\alpha}(u) d V+A[u]=\oint_{B} u^{a}\left(\frac{\partial u}{\partial \nu}\right)_{\alpha} d S
$$

where $\mathscr{A}_{\alpha}(u)$ is the operator consisting of the first set of terms on the right of (7.1), and

$$
A[u]=\iint_{D} a_{\alpha \beta}^{i j} u_{, i}^{\alpha} u_{, j}^{\beta} d V .
$$

In the case of the equations of elasticity, $(\partial u / \partial \nu)_{\alpha}$ is the surface traction, while $A[u]$ is the strain energy. In analogy with (2.12), one finds that if one defines

$$
\left(\frac{\partial u}{\partial t}\right)^{2}=a_{\alpha \beta}^{i j} u_{, i}^{\alpha} u_{, j}^{\beta}-\left[a_{\alpha \beta}^{i j} n_{i} n_{j}\right]^{-1}\left(\frac{\partial u}{\partial \nu}\right)_{\alpha}\left(\frac{\partial u}{\partial \nu}\right)_{\beta}
$$

where []$^{-1}$ denotes matrix inversion, then $(\partial u / \partial t)^{2}$ is a positive semidefinite quadratic form in the tangential derivatives of the components $u^{a}$. If $u^{a}$ vanishes on $B$, the left-hand side vanishes. If $u^{a}$ is given on $B$, the left-hand side may be computed by using another vector field $\bar{u}$ having the same values on $B$. Similarly, in analogy with (2.13) we have 


$$
f^{i} u_{, i}^{\alpha}=f^{i} n_{i}\left[a_{\alpha \beta}^{j k} n_{j} n_{k}\right]^{-1}\left(\frac{\partial u}{\partial \nu}\right)_{\beta}+f^{i} T_{i}^{\alpha} \frac{\partial u}{\partial t}
$$

where $T_{i}^{\alpha}$ is a matrix with the property

$$
a_{\alpha \beta}^{i j} T_{i}^{\alpha} T_{j}^{\beta}=1 \text {. }
$$

With these decompositions and the identity analogous to (2.3), one finds the analogue of (2.14), namely

$$
\begin{gathered}
\oint_{B}\left\{f^{k} n_{k}\left(\left(\frac{\partial u}{\partial t}\right)^{2}-\left[a_{\alpha \beta}^{j k} n_{j} n_{k}\right]^{-1}\left(\frac{\partial u}{\partial \nu}\right)_{\alpha}\left(\frac{\partial u}{\partial \nu}\right)_{\beta}\right)-2 f^{i} T_{i}^{\alpha}\left(\frac{\partial u}{\partial \nu}\right)_{\alpha} \frac{\partial u}{\partial t}\right\} d S \\
=-2 \iint_{D} f^{i} u_{, i}^{\alpha} \mathscr{A}_{\alpha}(u) d V+\iint_{D}\left[f_{, k}^{k} a_{\alpha \beta}^{i j}-2 f_{, k}^{i} a_{\alpha \beta}^{j k}+f^{k} a_{\alpha \beta, k}^{i j}\right] u_{, i}^{\alpha} u_{, j}^{\beta} d V .
\end{gathered}
$$

One may write this in terms of the operator $\mathscr{B}_{a}$ by means of the relation

$$
\mathscr{A}_{\alpha}(u)=\mathscr{B}_{\alpha}(u)+b_{\alpha \beta}^{i} u_{, i}^{\beta}+q_{\alpha \beta} u^{\beta} .
$$

It follows from (7.3) that $\left[a_{\alpha \beta}^{j k} n_{j} n_{k}\right]^{-1}$ is positive definite. Thus, one can proceed as in $\S 3$ to estimate the quadratic form $A[u]$ or

$$
B[u]=\iint_{D}\left[a_{\alpha \beta}^{i j} u_{, i}^{\alpha} u_{,{ }_{j}}^{\beta}+b_{\alpha \beta}^{i} u^{a} u_{, i}^{\beta}+q_{\alpha \beta} u^{a} u^{\beta}\right] d V .
$$

In order to get pointwise estimates, one uses the representation

$$
\begin{aligned}
& w^{a}(p)-\varphi^{a}(p)=\iint_{D}\left[\Gamma_{p}^{(\alpha) \beta} \mathscr{B}_{\beta}(w-\varphi)-\left(w^{\beta}-\varphi^{\beta}\right) \mathscr{B}_{\beta}^{*}\left(\Gamma_{p}^{(\alpha)}\right)\right] d V \\
& \quad+\oint_{B}\left[\left(w^{\beta}-\varphi^{\beta}\right)\left(\frac{\partial \Gamma_{p}^{(\alpha)}}{\partial \nu}\right)_{\beta}+\Gamma_{\rho}^{(\alpha) \rho} b_{\beta \rho}^{i} n_{i}-\Gamma_{\rho}^{(\alpha) \beta}\left(\frac{\partial}{\partial \nu}(w-\varphi)\right)_{\beta}\right] d S .
\end{aligned}
$$

Here

$$
\mathscr{B}_{\alpha}^{*}(u)=\left(\alpha_{\alpha \beta}^{i j} u_{, i}^{\beta}\right)_{, j}+\left(b_{\beta \alpha}^{i} u^{\beta}\right)_{, i}-q_{\beta \alpha} u^{\beta},
$$

and $\Gamma_{p}^{(\alpha) \beta}(x)$ is, for fixed $\alpha$ and point $p$ an $M$-vector field with properties analogous to the properties (4.1) of $\Gamma_{p}(x)$. The most important of these is the presence of a singularity at $p$ such that if $S_{\rho}$ is a sphere of radius $\rho$ centered at $p$,

$$
\lim _{\rho \rightarrow 0} \oint_{S_{\rho}}\left(\frac{\partial \Gamma_{p}^{(\alpha)}}{\partial \nu}\right)_{\beta} d S=\delta_{\beta}^{\alpha} .
$$

For the construction of such a parametrix tensor see [8].

The results of $\$ 5$ can be extended to the exterior problem with values of $(\partial u / \partial \nu)_{\alpha}+h_{\alpha \beta} u^{\beta}$ given on the boundary $B$. Here $h_{\alpha \beta}$ is a given positive semidefinite matrix field on $B$. 
Let the inverse of the matrix $\alpha_{\alpha \beta}^{i j}$ be denoted by $a_{i j}^{\alpha \beta}$ so that

$$
a_{\alpha \beta}^{i j} a_{j k}^{\beta \gamma}=\delta_{k}^{i} \delta_{\alpha}^{\gamma} .
$$

Let $g_{\alpha \beta}^{i}$ be a tensor field in the exterior $D$ of $B$ with

$$
G_{\alpha \beta}=g_{\alpha \beta, i}^{i}-g_{\alpha r}^{i} \alpha_{i j}^{r \delta} g_{s \beta}^{j}
$$

positive definite in $D$, and

$$
\tilde{G}_{\alpha \beta}=-g_{\alpha \beta}^{i} \nu_{i}+h_{\alpha \beta}
$$

positive definite on $B$. The analogue of (5.8) is

$$
\iint_{D} G_{\alpha \beta} u^{a} u^{\beta} d V+\oint_{B} \tilde{G}_{\alpha \beta} u^{a} u^{\beta} d S \leqq A[u]+\oint_{B} h_{\alpha \beta} u^{a} u^{\beta} d S .
$$

From this follows the inequality

$$
\begin{aligned}
A[u]+\oint_{B} h_{\alpha \beta} u^{a} u^{\beta} d S & \leqq \iint_{D} G^{a \beta} \mathscr{X}_{\alpha}(u) \mathscr{X}_{\beta}(u) d V \\
& +\oint_{B} \tilde{G}^{a \beta}\left[\left(\begin{array}{c}
\partial u \\
\partial \nu
\end{array}\right)_{\alpha}+h_{\alpha \gamma} u^{\gamma}\right]\left[\left(\frac{\partial u}{\partial \nu}\right)_{\beta}+h_{\beta \delta} u^{\delta}\right] d S,
\end{aligned}
$$

where $G^{\alpha \beta}$ and $\tilde{G}^{\alpha_{\beta}}$ are the inverse matrices of $G_{\alpha \beta}$ and $\tilde{G}_{\alpha \beta}$. This is the analogue of (5.11) and, together with (7.19), gives the analogue of (5.10). Thus we can proceed as in $\S 5$ to approximate the solution of the problem where $\mathscr{A}_{\circ}(u)$ is given in $D$ and $(\partial u / \partial \nu)_{\alpha}+h_{\alpha \beta} u^{\beta}$ is given on $B$. These results may be extended as outlined in $\S 6$ to the more general operator $\mathscr{B}_{\alpha}(u)$.

More generally, we may divide $B$ into components $B_{0}, B_{1}, B_{2}, \cdots, B_{n}$. On $B_{0}$ we prescribe $\left(\partial u / \partial_{\nu}\right)_{\alpha}+h_{\alpha \beta} u^{\beta}$. On $B_{k}$ we are given $k$ linearly independent vector fields. Then $u$ is required to be orthogonal to these $k$ vectors, while $(\partial u / \partial \nu)_{\alpha}+h_{\alpha \beta} u^{\beta}$ is prescribed to within an arbitrary linear combination of the $k$ vectors on $B_{k}$. As a special case we obtain the mixed boundary value problem of the theory of elasticity [13] where the displacement in certain directions and the components of force in the other directions are given.

It is clearly only necessary for the quadratic form $\widetilde{G}_{\alpha \beta} u^{\alpha} u^{\beta}$ with $u^{\alpha}$ subject to the orthogonality conditions on each component $B_{k}$ to be positive. Then the quadratic form in the second term on the right of (7.20) is defined, positive and known when the arbitrary linear combination of the $k$ vectors on $B_{k}$ is so chosen that $(\partial u / \partial \nu)_{\alpha}+h_{\alpha \beta} u^{\beta}$ satisfies the same orthogonality relations as $u^{\alpha}$.

The approximation proceeds from $(7.20)$ as in $\S 5$. 
Added in Proof. In the special case of the mixed boundary value problem when the given values of $w$ and the approximating function $\varphi$ on $B_{1}$ are zero or when $B_{2} \equiv B$, the estimate (5.12) may be replaced by the identity

$$
\begin{gathered}
A[w]+\int_{B_{2}} h w^{2} d S-\left\{-2 \iint_{D} \varphi \mathfrak{A}(w) d V+2 \int_{B_{2}} \varphi\left(\frac{\partial w}{\partial \nu}+h w\right) d S\right. \\
\left.\quad-A[\varphi]-\int_{B_{2}} h \varphi^{2} d S\right\} \\
=A[u]+\int_{B_{2}} h u^{2} d S
\end{gathered}
$$

which gives an error bound quadratic in $u$.

\section{REFERENCES}

1. R. Courant and D. Hilbert, Methoden der mathematischen Physik, Berlin (1937).

2. J.B. Diaz, Upper and lower bounds for quadratic functionals, Proc. Symp. Spectral Theory and Diff. Probs., Oklahoma A. and M. (1950), 279-289; see also paper with same title in Collectanea Math. 4 (1951), 3-50.

3. J. B. Diaz and H. Greenberg, Upper and lower bounds for the solution of the first boundary value problem of elasticity, Quart. Appl. Math. 6 (1948), 326-331.

4. G. Faber, Beweis dass unter allen homogenen Membranen von gleicher Fläche und gleicher Spannung die Kreisförmige den tiefsten Grundton gibt, Sitzungsb. Bayr. Akad. Wiss. (1923), 169-172.

5. G. Fichera, Alcuni recenti sviluppi della teoria dei problemi al contorno per le equazioni alle derivate parziali lineari, Atti del Convegno Internazionale sulle Equazioni alle Derivate Parziali; Trieste (1954).

6. P. Garabedian and M. Schiffer, Convexity of domain functionals, J. d'Analyse Math. 2 (1952-3), 281-368; esp. pp. 299-301.

7. L. Hörmander, Uniqueness theorems and estimates for normally hyperbolic partial differential equations of the second order, Comptes rendus du Douzième Congres des Mathématiciens Scandinaves Tenu á Lund (1953), 105-115.

8. F. John, Plane waves and spherical means applied to partial differential equations, Interscience Tracts No. 2 (1955).

9. E. Krahn, Uber eine von Rayleigh formulierte minimaleigenschaft des Kreises, Math. Ann. 94 (1924), 97-100.

10. L. E. Payne and H.F. Weinberger, New bounds in harmonic and biharmonic problems, J. Math. Phys. 4 (1955), 291-307.

11. L. E. Payne and H.F. Weinberger, New bounds for solutions of second order elliptic partial differential equations, BN-108 AF 18 (600)-573, University of Maryland (1957).

12. F. Rellich, Darstellung der Eigenwerte von $\Delta u+\lambda u$ durch ein Randintegral, Math. Z. 46 (1940), 635-646.

13. I. S. Sokolnikoff, Mathematical theory of elasticity, McGraw-Hill (1946).

14. J. L. Synge, Upper and lower bounds for the solutions of problems in elasticity, Proc. Roy. Irish Acad. 53 \#4 (1950) 41-64. 



\section{PACIFIC JOURNAL OF MATHEMATICS}

\section{EDITORS}

David Gilbarg

Stanford University

Stanford, California

R. A. Beaumont

University of Washington

Seattle 5, Washington

\author{
A. L. Whiteman
}

University of Southern California

Los Angeles 7, California

E. G. Straus

University of California

Los Angeles 24, California

\section{ASSOCIATE EDITORS}

\author{
E. F. BECKENBACH \\ C. E. BURGESS \\ M. HALL \\ E. HEWITT
}

\author{
A. HORN \\ V. GANAPATHY IYER \\ R. D. JAMES \\ M. S. KNEBELMAN
}

L. NACHBIN
I. NIVEN
T. G. OSTROM
H. L. ROYDEN

M. M. SCHIFFER

G. SZEKERES

F. WOLF

K. YOSIDA

\section{SUPPORTING INSTITUTIONS}

\author{
UNIVERSITY OF BRITISH COLUMBIA \\ CALIFORNIA INSTITUTE OF TECHNOLOGY \\ UNIVERSITY OF CALIFORNIA \\ MONTANA STATE UNIVERSITY \\ UNIVERSITY OF NEVADA \\ OREGON STATE COLLEGE \\ UNIVERSITY OF OREGON \\ UNIVERSITY OF SOUTHERN CALIFORNIA
}

\author{
STANFORD UNIVERSITY \\ UNIVERSITY OF UTAH \\ WASHINGTON STATE COLLEGE \\ UNIVERSITY OF WASHINGTON \\ AMERICAN MATHEMATICAL SOCIETY \\ CALIFORNIA RESEARCH CORPORATION \\ HUGHES AIRCRAFT COMPANY \\ THE RAMO-WOOLDRIDGE CORPORATION
}

Mathematical papers intended for publication in the Pacific Journal of Mathematics should be typewritten (double spaced), and the author should keep a complete copy. Manuscripts may be sent to any of the editors. All other communications to the editors should be addressed to the managing editor, E. G. Straus at the University of California, Los Angeles 24, California.

50 reprints per author of each article are furnished free of charge; additional copies may be obtained at cost in multiples of 50 .

The Pacific Journal of Mathematics is published quarterly, in March, June, September, and December. The price per volume (4 numbers) is $\$ 12.00$; single issues, $\$ 3.50$. Back numbers are available. Special price to individual faculty members of supporting institutions and to individual members of the American Mathematical Society: $\$ 4.00$ per volume; single issues, $\$ 1.25$.

Subscriptions, orders for back numbers, and changes of address should be sent to Pacific Journal of Mathematics, 2120 Oxford Street, Berkeley 4, California.

Printed at Kokusai Bunken Insatsusha (International Academic Printing Co., Ltd.), No. 6, 2-chome, Fujimi-cho, Chiyoda-ku, Tokyo, Japan.

\section{PUBLISHED BY PACIFIC JOURNAL OF MATHEMATICS, A NON-PROFIT CORPORATION}

The Supporting Institutions listed above contribute to the cost of publication of this Journal, but they are not owners or publishers and have no responsibility for its content or policies. 


\section{Pacific Journal of Mathematics}

\section{Vol. 8, No. 3 \\ May, 1958}

Michael Israel Aissen, A set function defined for convex plane domaines... . 383

Robert Ellis, Distal transformation groups ................... 401

Ciprian Foias, On a commutative extension of a commutative Banach algebra ....................................... 407

Jerry William Gaddum, Linear inequalities and quadratic forms ......... 411

Allen A. Goldstein and Elliott Ward Cheney, Jr., A finite algorithm for the solution of consistent linear equations and inequalities and for the Tchebycheff approximation of inconsistent linear equations...........

William L. Hart and T. S. Motzkin, Proof of the fundamental theorem on implicit functions by use of composite gradient corrections .......... 429

Henry Berge Helson, Conjugate series and a theorem of Paley .......... 437

Wu-Chung Hsiang, Abelian groups characterized by their independent subsets....................................... 447

John W. Lamperti, On the isometries of certain function-spaces ........ 459

Karel DeLeeuw and Walter Rudin, Extreme points and extremum problems

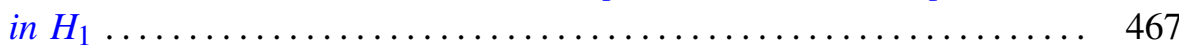

Eugene Lukacs, Some extensions of a theorem of Marcinkiewicz........ 487

George W. Mackey, Multiplicity free representations of finite groups ..... 503

Eben Matlis, Injective modules over Noetherian rings ............. 511

John William Neuberger, Continuous products and nonlinear integral equations

Lawrence Edward Payne and Hans F. Weinberger, New bounds for solutions of second order elliptic partial differential equations...

William T. Reid, A Prüfer transformation for differential systems ........ 575

Howard L. Rolf, The free lattice generated by a set of chains ...

K. M. Saksena, Inversion and representation theorems for a generalized

Laplace integral....................................... 597

Daniel Shanks, Two theorems of Gauss......................... 609

Paul Slepian, On the Lebesgue area of a doubled map ............... 613

Otto Szász and Nelson Paul Yeardley, Jr., The representation of an analytic function by general Laguerre series . ..................... 621

Alan C. Woods, On two-dimensional convex bodies ................. 635 